Int. J. Dev. Biol. 54: 33-39 (2010)

doi: $10.1387 / \mathrm{ijdb} .082802 \mathrm{~mm}$

\title{
A stem cell-derived gene (Sddr) negatively regulates differentiation of embryonic stem cells
}

\author{
MIWAKO MIURA ${ }^{1,2}$, ATSUSHI UEDA ${ }^{1}$, YUKINARI TAKAO ${ }^{1}$, EMI K. NISHIMURA², \\ HIROSHI KOIDE*,1 and TAKASHI YOKOTA ${ }^{1}$ \\ ${ }^{1}$ Department of Stem Cell Biology, Graduate School of Medical Science and \\ ${ }^{2}$ Department of Stem Cell Medicine, Cancer Research Institute, Kanazawa University, Kanazawa, Japan
}

\begin{abstract}
Embryonic stem (ES) cells, derived from the inner cell mass of blastocysts, are pluripotent and continue to self-renew. To better understand the molecular mechanisms underlying self-renewal, we have been searching for a gene(s) which is specifically expressed in selfrenewing ES cells. Here we report the isolation and characterization of a novel gene, Sddr (stem cell-derived differentiation regulator). Sddr was highly expressed in undifferentiated ES cells, and its expression was downregulated upon differentiation. In addition to ES cells, Sddr expression was observed strongly in ovary, and weakly in lung. Immunostaining and cellular fractionation analyses suggested that Sddr is a cytoplasmic protein associated with the cytoskeleton. Sddr-null ES cells showed no remarkable abnormalities in their undifferentiated state. In contrast, in differentiating Sddr-null cells, induction of several differentiation-associated markers was enhanced, and downregulation of self-renewal marker genes was accelerated, as compared with wild-type cells. These results suggest that although it is dispensable for ES cell self-renewal, Sddr is a negative regulator of ES cell differentiation.
\end{abstract}

KEY WORDS: ES cell, STAT3, Oct3/4, Sddr, self-renewal, differentiation, ovary

\section{Introduction}

Embryonic stem (ES) cells were established from inner cell mass (ICM) of mammalian blastocysts (Evans and Kaufman, 1981; Martin, 1981). Mouse ES cells require leukemia inhibitory factor (LIF) for self-renewal (Smith et al., 1988; Williams et al., 1988). LIF, which belongs to the interleukin- 6 cytokine family, acts through a receptor complex composed of a low-affinity LIF receptor and gp130 (Hibi et al., 1990; Gearing et al., 1991). We previously reported that the tyrosine residue of gp130 responsible for STAT3 activation is necessary for self-renewal in mouse ES cells (Matsuda et al., 1999). Using a fusion protein between STAT3 and the ligand-binding domain of estrogen receptor (STAT3ER), we also demonstrated that STAT3 activation is sufficient to maintain the undifferentiated state of ES cells. Furthermore, it has been shown that suppression of STAT3 activity results in differentiation of ES cells (Niwa et al., 1998). These observations indicate that STAT3 plays a critical role in the selfrenewal of mouse ES cells.

Oct3/4 and Nanog are other important transcription factors for self-renewal of ES cells. Oct3/4 is a POU transcription factor expressed in early embryo cells and germ cells. Oct3/4-deficient embryos fail to form ICM (Nichols et al., 1998). In ES cells, a reduced level of Oct3/4 leads to trophoectodermal differentiation, while its overexpression induces differentiation into primitive endoderm and mesoderm (Niwa et al., 2000), indicating that Oct3/ 4 is indispensable for ES cell self-renewal. Nanog is a homeoprotein that has been identified as a self-renewal-promoting gene (Chambers et al., 2003) and as a gene specifically expressed in ES cells (Mitsui et al., 2003). Constitutive expression of Nanog enables self-renewal of ES cells even in the absence of LIF, and Nanogdeficient ICM failed to generate epiblast and produced only parietal endoderm-like cells, suggesting the importance of Nanog in ES cell self-renewal. A recent report, however, has demonstrated that Nanog-deficient ES cells can self-renew indefinitely, although showing the tendency to differentiate (Chambers et al.,

\footnotetext{
Abbreviations used in this paper: 4HT, 4-hydroxytamoxifen; ES, embryonic stem; ICM, inner cell mass; LIF, leukemia inhibitory factor; Sddr, stem cellderived differentiation regulator; STAT3ER, fusion protein between STAT3 and the ligand-binding domain of estrogen receptor; Tet, tetracycline.
}

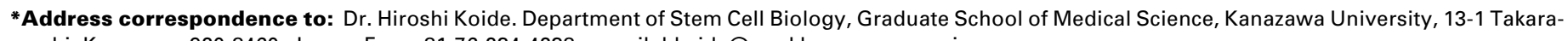
machi, Kanazawa 920-8460, Japan. Fax: +81-76-234-4238. e-mail: hkoide@med.kanazawa-u.ac.jp 
A

\begin{abstract}
MASHTADADA KPDSDSQKLL NVLPVSLRLR TRPWWFPIQE VSNPLVLYME AWVAERVIGT DQAEISEIEW MCQALLTVDS VNSGNLAEIT IFGQPSAQTR MKNILLNMAA WHKENELQRA VKVKEVEEFL KIRASSILSK LSKKGLKLAG FPLPLEGRET QMES
\end{abstract}
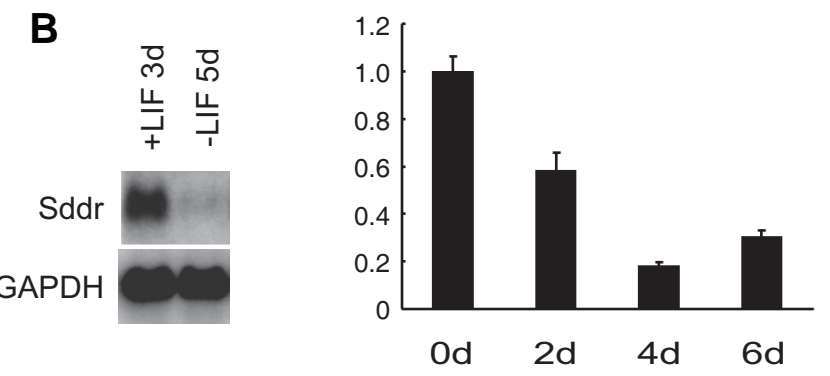

Fig. 1. Sddr is specifically expressed in self-renewing ES cells. (A) Amino acid sequence of Sddr. (B) Downregulation of Sddr mRNA during ES differentiation. (Left panel) ES cells were cultured with LIF for three days or without LIF for five days and subjected to Northern blot analysis. GAPDH was used as an internal control (Willems et al., 2006). (Right panel) $E S$ cells were cultured in the absence of LIF for the indicated days and subjected to real-time PCR. The value at day 0 is set to 1.0. Bars represent the means and standard errors of triplicates.

A
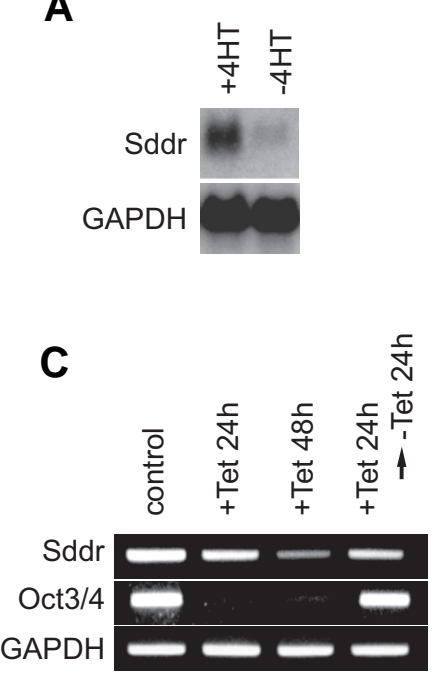
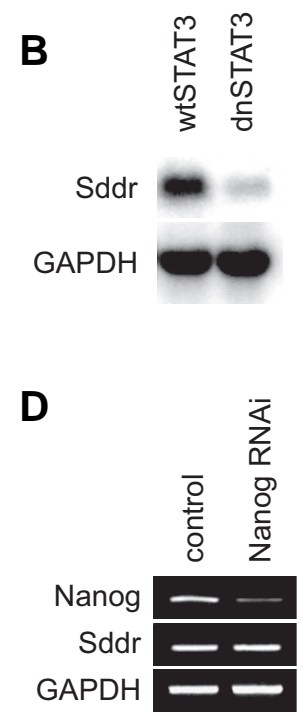

2007). These observations suggest that Nanog is a promoting factor, rather than an indispensable factor, in ES cell self-renewal.

In this study, to understand the molecular mechanism of selfrenewal in ES cells, we searched for a gene(s) specifically expressed in self-renewing ES cells, and we isolated Sddr (stem cell-derived differentiation regulator). Although disrupting this gene had no effect on the maintenance of ES cells, it promoted ES cell differentiation, suggesting that Sddr regulates a switching between self-renewal and differentiation in ES cells.

\section{Results}

\section{Identification of Sddr as a self-renewing ES-specific gene}

To identify self-renewal-specific genes, we performed microarray analysis to search for a gene(s) whose expression is upregulated by both LIF stimulation and STAT3ER activation, and found 2410146L05Rik, which encodes a novel protein of 164 amino acids with no known motif (Fig. 1A). We named this gene Sddr (stem cell-derived differentiation regulator) to reflect its properties: the gene is highly expressed in self-renewing ES cells and is involved in regulating ES cell differentiation (see below).

The expression of Sddr in self-renewing ES cells was verified by Northern blot analysis and quantitative RT-PCR (Fig. 1B). Robust expression of Sddr was detected in ES cells cultured with LIF, while LIF removal led to quick downregulation of this expression. STAT3ER-expressing ES cells possessed a higher level of Sddr in the presence of $4 \mathrm{HT}$ compared with those cultured without 4HT (Fig. 2A). ES cells transfected with a dominant-negative mutant of STAT3 showed a lower expression level of Sddr compared with cells transfected with wild-type STAT3 (Fig. 2B).
A

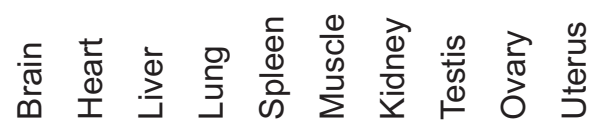

Sddr

GAPDH

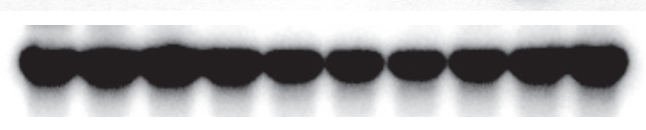

B

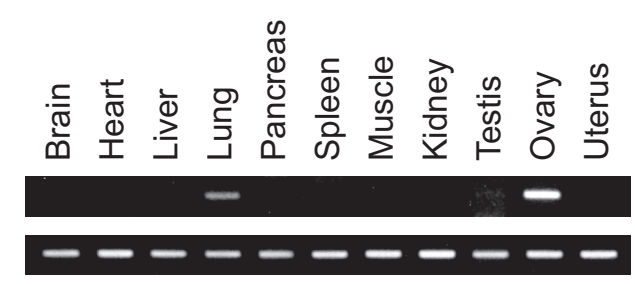

Fig. 2. Relationship of Sddr with STAT3, Oct3/4 and Nanog (Left). (A) STAT3ER-expressing ES cells were incubated with (+) or without (-) 4HT for four days. (B) ES cells were transfected with either wild-type STAT3 (wtSTAT3) or dominant-negative mutant of STAT3 (dnSTAT3) and selected for two days in the presence of puromycin $(1 \mu \mathrm{g} / \mathrm{ml})$. Cells were cultured for two more days in the absence of puromycin and harvested. In each experiment, total RNA (10 $\mathrm{gg}$ ) was loaded to $1.5 \%$ agarose gel and subjected to Northern blot analysis. (C) ZHBTc4 cells were incubated with (+) or without (-) tetracycline (Tet) for 24 or $48 \mathrm{~h}$ and subjected to RT-PCR analysis. Control, ZHBTc4 cells maintained in the absence of Tet. (D) RNAiconstruct for Nanog (Nanog RNAi) or an empty vector, pSi-puro (control), was introduced into ES cells. After $24 \mathrm{~h}$ culture, cells were transferred to new dishes and incubated for another $24 \mathrm{~h}$, and the medium was exchanged for a new medium containing $1 \mu \mathrm{g} / \mathrm{ml}$ puromycin. Cells were cultured for three more days and subjected to RT-PCR analysis.

Fig. 3. Tissue distribution of Sddr (Right). Expression of Sddr in the indicated tissue was examined by Northern blot analysis (A) and RT-PCR analysis (B). 


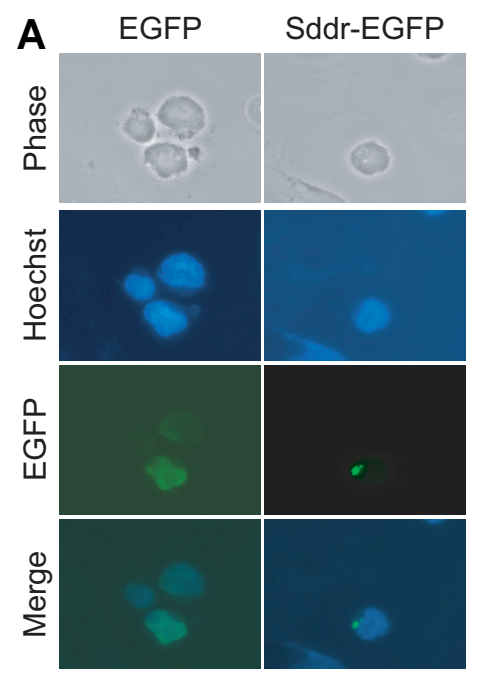

B
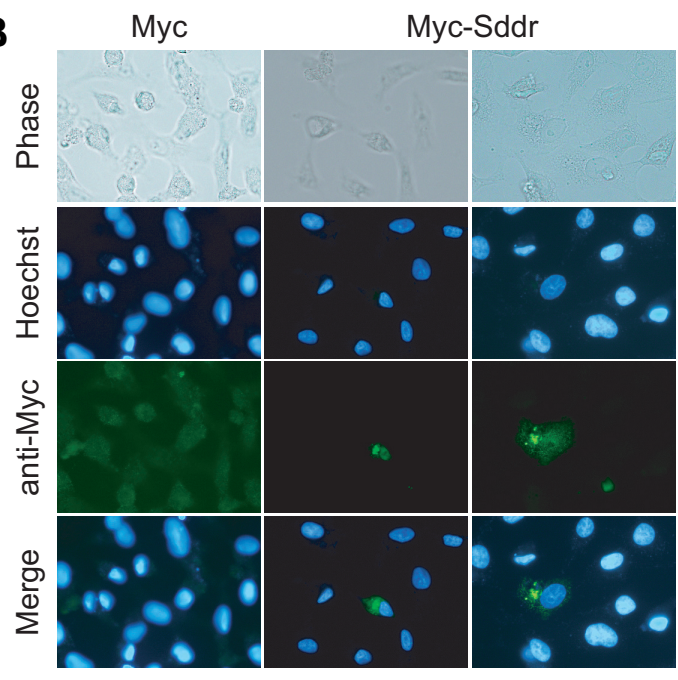

C

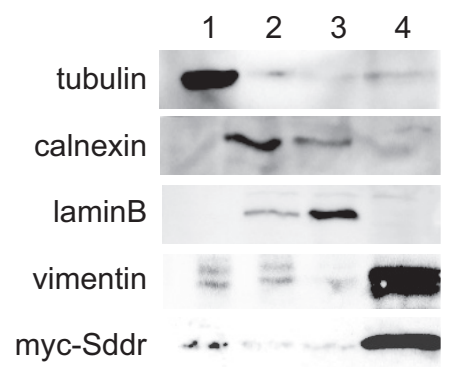

Fig. 4. Localization of Sddr in ES cells. (A) $E S$ cells were transfected with pEGFP-N2 (EGFP) or pEGFP-N2-Sddr (Sddr-EGFP), and cultured for $24 \mathrm{~h}$. Cells were pelleted onto glass slides using a Cytospin and analyzed with a fluorescence microscope. (B) HeLa cells were transfected with pCAG-myc-IP (Myc) or pCAG-myc-Sddr-IP (Myc-Sddr) and cultured for two days. Myc-Sddr was detected by antimyc antibody. (C) Western blot analysis of cellular fractions from myc-Sddr-transfected ES cells. Lane 1, cytoplasm; lane 2, membrane/organella; lane 3, nucleus; lane 4, cytoskeleton. Tubulin, calnexin, laminB, and vimentin were used as markers for cytoplasm, membrane/organella, nucleus, and cytoskeleton fractions, respectively. Myc-Sddr was detected with an anti-myc antibody.

expression of Sddr-EGFP was driven by the CAG promoter, this protein localized in the cytoplasm of ES cells (Fig. 4A). Cytoplasmic localization of Sddr is also observed in HeLa cells expressing myctagged Sddr (Fig. 4B). Furthermore, cellular fractionation analysis revealed that, in ES cells, Sddr exists in the cytoskeleton fraction together with a cytoskeleton marker, vimentin (Fig. 4C). These data suggest that Sddr is localized in the cytoplasm of ES cells and is associated with the cytoskeleton.

\section{Targeted disruption of the sddr gene in ES cells}

Since Sddr is expressed in undifferentiated ES cells, it is possible that Sddr is involved in the maintenance of ES cell self-renewal. To explore this possibility, we established Sddr-null ES cells with two targeting constructs to replace the entire open-reading frame region with the $\beta$-galactosidase (LacZ) and neomycinresistance (neo) genes or hygromycinresistance (hygro) gene (Fig. 5A). The LacZ-neo targeting vector was introduced into ES cells by electroporation, and neomycin-resistant cells were selected in medium containing G418. Of 360 G418-resistant clones screened, four clones were isolated as Sddr+/- clones. The homologous recombinations in all clones were

When we examined the expression level of Sddr in ZHBTc4 ES cells, in which Oct3/4 expression can be artificially regulated by addition of tetracycline (Tet) (Niwa et al., 2000), Oct3/4 and Sddr were strongly expressed (Fig. 2C). As Oct3/4 expression was downregulated by addition of Tet, Sddr expression gradually decreased. When Oct3/4 expression was recovered by removal of Tet, downregulation of Sddr mRNA ceased, but was not restored. When we suppressed expression of Nanog by RNA interference (RNAi), the expression level of Sddr was not changed despite Nanog downregulation (Fig. 2D). Taken together, the results indicate that Sddr is a self-renewal-specific gene, and suggest the possibility that expression of Sddr may be regulated by STAT3 and Oct3/4.

\section{Expression of Sddr in adult tissues}

To examine whether the expression of Sddr is restricted to ES cells, we prepared total RNAs from several adult mouse tissues and performed Northern blot analysis. As shown in Fig. 3A, Sddr transcript was strongly expressed in ovary, which corresponds well with the recent report by Joshi et al. (2007). In addition, RTPCR analysis suggested that Sddr is expressed also in lung (Fig. 3B).

\section{Sddr is localized in the cytoplasm}

To determine the localization of Sddr in ES cells, we constructed Sddr fusion protein with EGFP (Sddr-EGFP). When the confirmed by PCR analysis (Fig. 5B), Southern blotting (Fig. $5 \mathrm{C}$ ), and X-gal staining (data not shown). To obtain homozygous mutant ES cells, we next introduced the hygro targeting vector into Sddr ${ }^{+/}$ES cells. Of 360 G418- and hygromycinresistant clones, two clones were isolated as $\mathrm{Sddr}^{-/-}$clones by PCR (Fig. 5B). In both clones (\#54-2, \#78-1), the homologous recombinations were confirmed by Southern blot analyses (Fig. 5C). Furthermore, Northern blot analysis showed that Sddr mRNA is absent in both clones (Fig. 5D).

\section{Sddr is dispensable for self-renewal, but it plays a negative role in regulation of differentiation in ES cells}

When we compared Sddr ${ }^{-/}$cells with the wild-type ES cells, we found that Sddr ${ }^{-/}$cells could be maintained with LIF and were normal in morphology (Fig. 6A), Oct3/4 and Nanog expression (Fig. 6B), cell-cycle structure (Fig. 6C), and proliferation (Fig. 6D). These results indicate that Sddr is dispensable for the self-renewal of ES cells. However, we noticed that the expression level of Gata4 was slightly higher in Sddr-null cells than in the wild-type cells (Fig. 6B), suggesting the possible involvement of Sddr in repressing ES cell differentiation. To assess this possibility, Sddr-- ES cells were subjected to in vitro differentiation through embryoid body formation. No apparent differences in the efficiency of forming embryoid bodies were observed between the wild-type and $\mathrm{Sddr}^{-/}$cells (data not shown). However, self-renewal marker genes, Oct3/4 and 
Nanog, were more quickly downregulated in differentiated Sddr-cells than in the wild-type cells (Fig. 6E). We next compared the expression levels of differentiation-associated markers between the wild-type and Sddr-null cells. Since we detected a slight upregulation of Gata4 in self-renewing Sddr-null cells, we first examined the effect of Sddr deficiency on induction of Gata4, and found that Gata4 is induced more strongly in Sddrnull cells. Similarly, induction of other endoderm markers, Gata6 and Sox17, was apparently accelerated in differentiated Sddr-null cells. Furthermore, Sddr deficiency also promoted induction of other germ layer markers, Fgf5 (ectoderm), Pax6 (neuroectoderm), T, Tbx5 (mesoderm), Cdx2 and Hand1 (trophectoderm). The observed phenotype was due to Sddr deficiency, because ectopic expression of Sddr suppressed downregulation of self-renewal markers, as well as induction of differentiation markers (Fig. 6F). These data suggest that

A

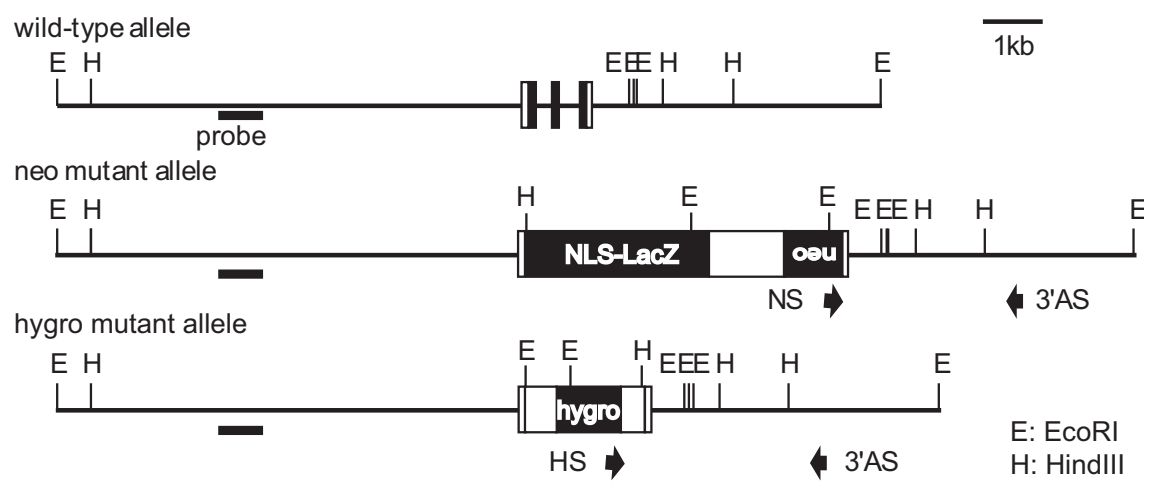

B

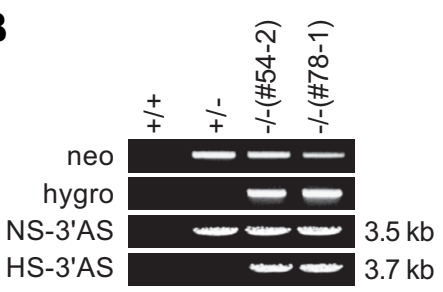

D

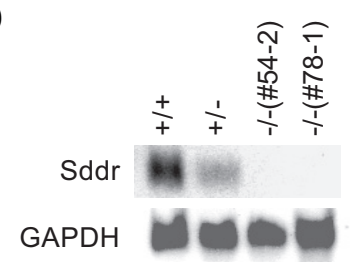

Fig. 5. Targeted disruption of the Sddr gene. (A) Schematic representations of the wildtype allele, targeted neo-mutant allele, and targeted hygro-mutant allele. The solid box in the wild-type allele represents the coding sequence. Arrows show oligonucleotide primers used in PCR screening. (B) Selection of heterozygous and homozygous ES cells by PCR with primers for neo and hygro genes, and mutant alleles (NS-3'AS, HS-3'AS). (C) Southern blot analysis of mutant clones using probe for the 5' region of the Sddr gene. (Upper panel) EcoRI digesta; wild-type allele $10.3 \mathrm{~kb}$, neo mutant allele $11.4 \mathrm{~kb}$, hygro mutant allele $8.4 \mathrm{~kb}$. (Lower panel) Hindlll digesta; wild-type allele $10.2 \mathrm{~kb}$, neo mutant allele $7.7 \mathrm{~kb}$, hygro mutant allele $9.7 \mathrm{~kb}$. (D) Northern blot analysis of Sddr transcripts. Total RNAs $(10 \mu \mathrm{g})$ were loaded to $1.5 \%$ agarose gel. Sddr mRNA was detected as a $0.8 \mathrm{~kb}$ band. +/+, wild-type cells; +/-, neo heterozygous mutant cells; --, homozygous mutant cells. disruption of the Sddr gene promotes differentiation of ES cells.

\section{Discussion}

Pluripotency is maintained during ES cell self-renewal through the prevention of differentiation and the promotion of proliferation. well-established that LIF is a key factor preventing differentiaa self-renewal-specific molecule in ES cells. Although known motif in Sddr protein, we inferred that Sddr is analysis indicated that Sddr is dispensable for self-renewal but does play a role in repression of differentiation in ES cells.

Since expression of Sddr is restricted to self-renewing ES cells, it is reasonable to assume that its expression is controlled by important transcription factors for self-renewal, such as STAT3, Oct3/4, and Nanog. Indeed, we demonstrated that suppression of STAT3 activity resulted in downregulation of Sddr, using the dominant-negative mutant of STAT3 and STAT3ER (Fig. 2A and B). However, since downregulation of STAT3 causes ES cell differentiation, we cannot determine whether downregulation of Sddr is due to inactivation of STAT3 or due to differentiation of ES cells. Therefore, although the present data suggest that Sddr is a putative target of STAT3, more detailed analysis, such as promoter analysis and chromatin immunoprecipitation assay, will be required to determine whether Sddr is a direct target of STAT3. As for the relationship between Sddr and Oct3/4, it seems that Oct3/4 indirectly regulates Sddr expression, since the influence of Oct3/4 repression on Sddr expression was quite small (Fig. 2C). In agreement with this conclusion, by combining the microarray data of ZHBTc4 cells with ChIP-chip and ChIPPET data, Matoba et al. (2006) have reported that 2410146L05Rik (Sddr) is not the primary but rather the secondary or even tertiary target of Oct3/4. As for Nanog, knockdown experiments suggested that this transcription factor is not involved in the regulation of Sddr expression, although we cannot exclude the possibility that suppression of Nanog might be insufficient to influence Sddr expression (Fig. 2D).

As compared with wild-type cells, the expression of Oct3/4 was reduced more rapidly in differentiating Sddr-null ES cells (Fig. 6E). Sddr deficiency also promoted downregulation of Nanog expression, as well as the inductions of multiple differentiation markers, during differentiation (Fig. 6E). Furthermore, we observed that Sddr-null ES cells produced more beating cells than parental ES cells when they underwent differentiation (data not shown). These results suggest that Sddr somehow controls a switching between self-renewal and differentiation in 
A

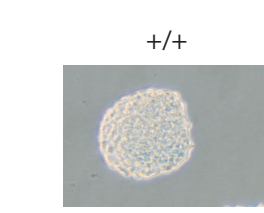

C

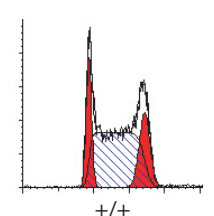

$\mathrm{G} 1: \mathrm{S}: \mathrm{G} 2 / \mathrm{M}=$

17.6:62.2:20.2
B
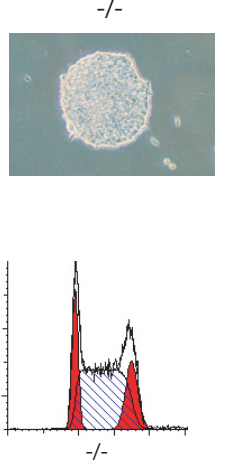

$\mathrm{G} 1: \mathrm{S}: \mathrm{G} 2 / \mathrm{M}=$

19.2:62.1:18.7

D
E

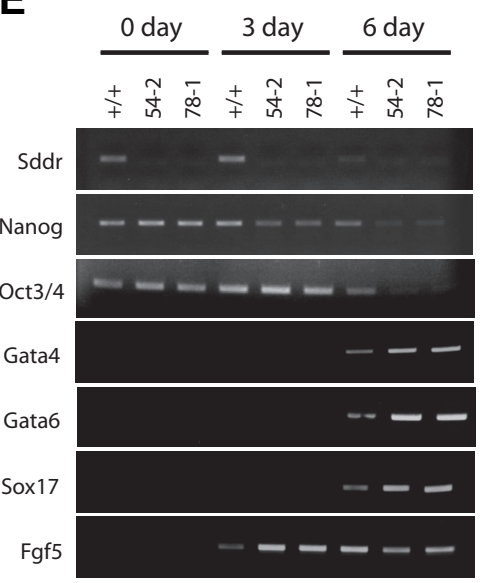

$\mathbf{F}$

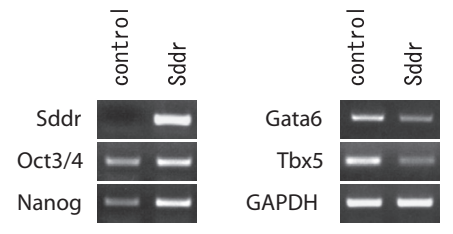

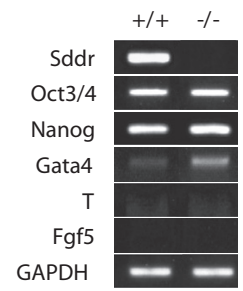
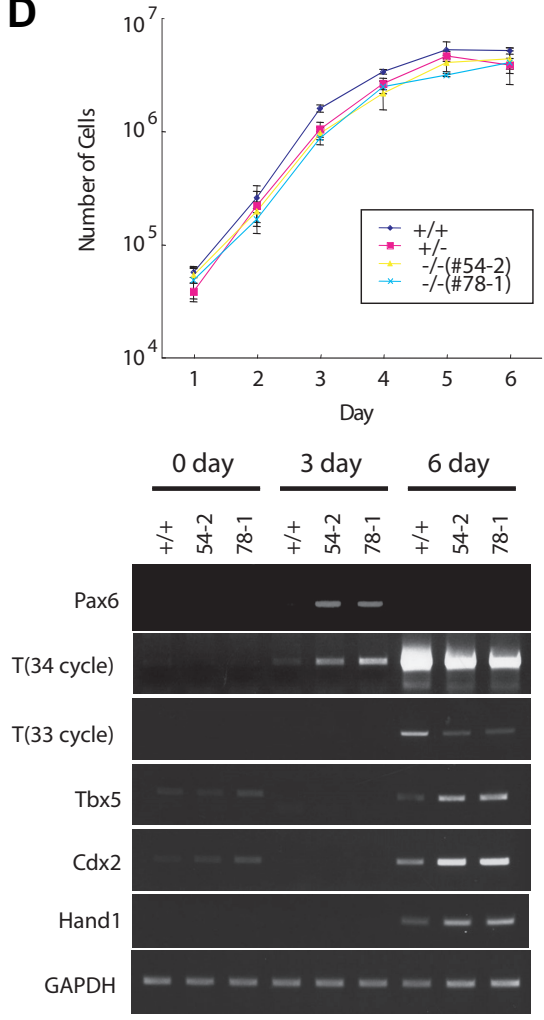

Fig. 6. Phenotypes of Sddr-null cells.

(A) Morphology of Sddr-null cells. Wildtype $(+/+)$ and Sddr-null cells (\#78-1) (-/-) were cultured with LIF for three days. (B) Expression levels of Sddr, Oct3/4, Nanog, Gata4, T and Fgf5 in Sddr-null ES cells. Wild-type $(+/+)$ and Sddr-null ES cells (\#78-1) (-/-) were cultured in the presence of LIF for three days, and the expression level of each gene was compared by RT-PCR. (C) Analysis of cell-cycle distribution. Wild-type $(+/+)$ and Sddr-null ES cells (\#78-1) (-/-) were cultured for $24 \mathrm{~h}$. The numbers represent the percentage of cells in the G1, S, and G2 to $M$ phases of the cell cycle. (D) Proliferation of Sddr-null ES cells. Cells were plated to a 6 -well plate at $3 \times 10^{4}$ cells perwell. Cell numbers were counted daily for six days. (E) Expression levels of selfrenewal and differentiation marker genes in differentiated Sddr-null ES cells. Wildtype (+/+) and Sddr-null ES cells (\#54-2 and \#78-1) were allowed to form embryoid bodies for the indicated days and subjected to RT-PCR analysis. (F) Suppression of accelerated differentiation of Sddr-null ES cells by ectopic expression of Sddr. Sddrnull ES cells (\#78-1) were transfected with pEFlaclP-hrGFP (control) or pEFlaclPsddr (Sddr), and stable clones were established by puromycin selection. Each clone was allowed to form embyoid bodies for six days and subjected to RT-PCR analysis. may imply a role of Sddr in other stem cells.

Our data suggest that Sddr localizes in the cytoplasm and associates with the cytoskeleton. How then does this protein regulate expression of differentiation-associated genes? Several studies have shown that cytoskeleton-associated proteins regulate transcription factors by trapping them in the cytoplasm. For example, Keap1, an actin-binding protein, controls the transactivity of Nrf2 by retaining Nrf2 in the cytoplasm (Itoh et al., 1999). LMP-4, which is associated with the actin cytoskeleton, indirectly regulates the transcription of Tbx5 target genes, Fgf10 and ANF, through trapping Tbx5 in the cytoplasm (Camarata et al., 2006). Therefore, it is possible that Sddr sequesters an essential molecule for ES cell differentiation in the cytoskeleton to prevent expression of differentiation-associated genes. Identification of an Sddr-interacting protein would provide a clue to understanding the role of Sddr in ES cells.

In conclusion, the present data suggest that Sddr regulates a switching step from self-renewal to differentiation in ES cells. Further analysis of Sddr may open up new insights for understanding how self-renewing ES cells are prevented from differentiation. In addition, Sddr may be an attractive target for regulation of ES cell differentiation in the future.

\section{Materials and Methods}

\section{Cell culture}

ES cell lines A3-1 (Azuma and Toyoda, 1991) and ZHBTc4 (Niwa et al., 2000) were maintained on gelatin-coated dishes in the absence of feeder cells, as described previously (Matsuda et al., 1999). STAT3ER-expressing ES cells were cultured with 4-hydroxytamoxifen (4HT, Sigma). HeLa cells were cultured in Dulbecco's modified Eagle medium containing $10 \%$ fetal bovine serum.

For in vitro differentiation, $1 \times 10^{6}$ ES cells were transferred to a $6-\mathrm{cm}$ petri dish and cultured without LIF to undergo embryoid body formation. Cells were harvested after three and six days.

\section{Plasmid construction and transfection}

Construction of expression vectors for the wild-type STAT3 (pCAG-wtSTAT3-IP) and dominant-negative mutant of STAT3 (pCAG-dnSTAT3-IP) was described previously (Akagi et al., 2005). The coding sequence of Sddr (GenBank accession number $A B 283026$ ) was amplified from cDNAs synthesized with total RNA of A3-1 cells using: sense (5'-TAA GAA TTC ACC ATG GCA TCC CAC ACG GCT GAT GC-3') and antisense (5'-TTA CGC CGG CGT TAA GAC TCC ATC TGT GTT TCT CTT C-3') oligonucleotide primers. pCAG-mycSddr-IP and $p E G F P-N 2-S d d r$ were constructed by inserting myc-tagged Sddr coding fragment into the mammalian expression vectors, pCAG-IP (Yoshida-Koide et al., 2004) and pEGFP-N2 (Clontech), respectively. pEFlaclP-hrGFP and pEFlaclP-sddr were constructed by inserting cDNAs of GFP and Sddr into pEFlacIP, respectively. pEFlacIP was produced by transferring a DNA fragment carrying internal ES cells. In addition, the present observation that Sddr is expressed also in ovary and lung (Fig. 3) suggests that this molecule may act as a differentiation regulator in types of cells other than ES cells. In particular, the expression of Sddr in female germ cells ribosome entry site (IRES) sequence and puromycin resistance gene from pCAG-IP into pEF-LACAB (Yamazaki et al., 2001). The target sequence (5'-GGT GCT TGC TTG TCC TTG G-3') of RNA interference for Nanog was cloned into the Apal and EcoRI sites of pSi-puro (Akagi et al., 
2005). ES cells were transfected by lipofection using LipofectAMINE 2000 (Invitrogen), and then selected with $1 \mu \mathrm{g} / \mathrm{ml}$ puromycin at $48 \mathrm{hr}$ posttransfection. HeLa cells were transfected by electroporation (240V, 500 $\mu \mathrm{F})$ using Gene Pulser II (Bio Rad).

\section{Northern blot, RT-PCR and real-time PCR analyses}

The total RNAs of individual cultured cells were extracted using Trizol Reagent. Northern blot analysis was carried out as previously described (Akagi et al., 2005). Probes corresponding to the entire coding regions of Sddr and glyceraldehyde-3-phosphate dehydrogenase (GAPDH) were labeled with $\left[\alpha^{32} \mathrm{P}\right] \mathrm{dCTP}$ using Megaprime DNA Labeling System (Amersham Biosciences).

For RT-PCR analysis, cDNA synthesis was performed with SuperScriptllI Reverse Transcriptase (Invitrogen) as described previously (Kajihara et al., 2003). Real-time PCR analysis was done with FullVelocity SYBR Green QPCR Master Mix (Stratagene) using the Mx3000p System (Stratagene). The amount of Sddr mRNA was determined from the appropriate standard curve and divided by the amount of GAPDH mRNA for normalization. Primers for Sddr were

5'-CTGGTGGTTCCCAATTCAGGAAGT-3' and

5'-CCTTCACAGCTCTTTGGAGTTCGT-3'. Primers for GAPDH, Oct3/4, Nanog, Gata4, Gata6, Sox17, Fgf5, Pax6, T (Brachyury), Tbx5, Cdx2, Hand1 were described before (Niwa et al., 2000; Yoshida-Koide et al., 2004; Akagi et al., 2005; Kinoshita et al., 2007; Ura et al., 2008).

\section{Immunostaining}

Immunostaining of cultured cells was carried out as previously described (Ogino and Yasuda, 1998). Briefly, the cells were fixed and incubated with mouse monoclonal anti-myc antibody (Santa Cruz Biotechnology), and then with Alexa Fluor 488 rabbit anti-mouse IgG (Molecular Probes). Hoechst (bisBENZIMIDE, Sigma) was added to final wash solution at the final concentration of $10 \mu \mathrm{g} / \mathrm{ml}$.

\section{Cellular fractionation and Western blot analysis}

A3-1 ES cells were transfected with pCAG-myc-Sddr-IP and harvested at $48 \mathrm{~h}$ post-transfection. Cellular fractions of the transfected cells were extracted using a Subcellular ProteoExtract Kit S-PEK (Calbiochem). Western blotting was carried out using mouse monoclonal anti- $\alpha$-tubulin (ICN), rabbit polyclonal anti-calnexin (H-70, Santa Cruz Biotechnology), goat polyclonal anti-laminB (M-20, Santa Cruz Biotechnology), goat polyclonal anti-vimentin (C-20, Santa Cruz Biotechnology), and mouse monoclonal anti-myc (Upstate) antibodies.

\section{Targeted disruption of Sddr}

The 5'-arm (4712 bp) and 3'-arm (2584 bp) were isolated by PCR. To construct the two gene-targeting vectors for Sddr locus, the neomycin phosphotransferase (neo) and LacZ genes, or phosphoglycerate kinase (PGK) promoter and the hygromycin resistance gene (hygro) were inserted into the Sddr open-reading frame (ORF) region. A diphtheria toxin A gene was inserted at the end of the 3' short arm of the targeting vectors for negative selection. Targeted ES cells were identified by PCR screening using Sddr antisense primer:

(3'AS: 5'-GCTAGGCCTGCATCATGGAGTTGTTGCTTC-3') in combination with neo sense

(NS: 5'-AGCAGCCGATTGTCTGTTGTGCCCAGTCAT-3')

or hygro sense

(HS: 5'-AGAAGTACTCGCCGATAGTGGAAACCGACG-3') primers. Independent clones, which had undergone homologous recombination at the Sddr locus, were isolated. Their genotypes were verified by Southern blot hybridization analyses using a partial 5' long arm region of Sddr (881 bp) as a probe, and by PCR for neo or hygro genes (Akagi et al., 2005). The 881-bp probe was isolated by PCR with 5'AACTCACAGAGATCTACTGC-3' and 5'-TACTCACTGAGTCTGGTCC3'.

\section{Acknowledgments}

We would like to thank Dr. T. Akagi and other members of our laboratory for their helpful discussions. We are grateful to Dr. H. Niwa (RIKEN Center for Developmental Biology, Japan) for providing ZHBTC4 cells. This work was supported in part by a Grant-in-Aid from the Ministry of Education, Culture, Sports, Science and Technology of Japan.

\section{References}

AKAGI, T, USUDA, M., MATSUDA, T., KO, M.S.H., NIWA, H., ASANO, M., KOIDE, $H$. and YOKOTA, T. (2005). Identification of Zfp-57 as a downstream molecule of STAT3 and Oct-3/4 in embryonic stem cells. Biochem. Biophys. Res. Commun. 331: 23-30.

AZUMA, S. and TOYODA, Y. (1991). Production of a germ-line chimeric mouse derived from newly established embryonic stem cells. Jpn. J. Anim. Reprod. 37: 37-43.

CAMARATA, T., BIMBER, B., KULISZ, A., CHEW, T.-L., YEUNG, J. and SIMON, H.-G. (2006). LMP4 regulates Tbx5 protein subcellular localization and activity. J. Cell Biol. 174: 339-348.

CHAMBERS, I., COLBY, D., ROBERTSON, M., NICHOLS, J., LEE, S., TWEEDIE, S. and SMITH, A. (2003). Functional expression cloning of Nanog, a pluripotency sustaining factor in embryonic stem cells. Cell 113: 643-655.

CHAMBERS, I., SILVA, J., COLBY, D., NICHOLS, J., NIJMEIJER, B., ROBERTSON, M., VRANA, J., JONES, K., GROTEWOLD, L. and SMITH, A. (2007). Nanog safeguards pluripotency and mediates germline development. Nature 450: 1230-1234.

EVANS, M.J. and KAUFMAN, M.H. (1981. Establishment in culture of pluripotential cells from mouse embryo. Nature 292: 154-6.

GEARING, D.P., THUT, C.J., VANDEBOS, T., GIMPEL, S.D., DELANEY, P.B., KING, J., PRICE, V., COSMAN, D. and BECKMANN, M.P. (1991). Leukemia inhibitory factor receptor is structurally related to the IL-6 signal transducer, gp130. EMBO J. 10: 2839-48.

HIBI, M., MURAKAMI, M., SAITO, M., HIRANO, T., TAGA, T. and KISHIMOTO, T. (1990). Molecular cloning and expression of an IL-6 signal transducer, gp130. Cell 63: 1149-1157.

ITOH, K., WAKABAYASHI, N., KATOH, Y., ISHII, T., IGARASHI, K., ENGEL, J.D. and YAMAMOTO, M. (1999). Keap1 represses nuclear activation of antioxidant responsive elements by Nrf2 through binding to the amino-terminal Neh2 domain. Genes Dev. 13: 76-86.

JOSHI, S., DAVIES, H., SIMS, L. P., LEVY, S. E. and DEAN, J. (2007). Ovarian gene expression in the absence of FIGLA, an oocyte-specific transcription factor. BMC Dev. Biol. 7: 67.

KAJIHARA, M., SONE, H., AMEMIYA, M., KATOH, Y., ISOGAI, M., SHIMANO, H., YAMADA, N. and TAKAHASHI, S. (2003). Mouse MafA, homologue of zebrafish somite Maf 1, contributes to the specific transcriptional activity through the insulin promoter. Biochem. Biophys. Res. Commun. 312: 831-842.

KINOSHITA, K., URA, H., AKAGI, T., USUDA, M., KOIDE, H. and YOKOTA, T. (2007). GABP $\alpha$ regulates Oct-3/4 expression in mouse embryonic stem cells. Biochem. Biophys. Res. Commun., 353: 686-691.

MARTIN, G.R. (1981). Isolation of a pluripotent cell line from early mouse embryo cultured in medium conditioned by teratocarcinoma stem cells. Proc. Natl. Acad. Sci. USA 78: 7634-7638.

MATOBA, R., NIWA, H., MASUI, S., OHTSUKA, S., CARTER, M.G., SHAROW, A.A. and KO, M.S.H. (2006). Dissecting Oct3/4-regulated gene networks in embryonic stem cells by expression profiling. PloS ONE 1: e26.

MATSUDA, T., NAKAMURA, T., NAKAO, K., ARAI, T., KATSUKI, M., HEIKE. and T., YOKOTA, T. (1999). STAT3 activation is sufficient to maintain an undifferentiated state of mouse embryonic stem cells. EMBO J. 18: 4261-4269.

MITSUI, K., TOKUZAWA, Y., ITOH, H., SEGAWA, K., MURAKAMI, M., TAKAHASHI, K., MARUYAMA, M., MAEDA, M. and YAMANAKA, S. (2003). The homeoprotein Nanog is required for maintenance of pluripotency in mouse epiblast and ES cells. Cell 113: 631-642.

NICHOLS, J., ZEVNIK, B., ANASTASSIADIS, K., NIWA, H., KLEWE-NEBENIUS D., CHAMBERS, I., SCHOLER, H. and SMITH, A. (1998). Formation of pluripotent stem cells in the mammalian embryo depends on the POU transcrip- 
tion factor Oct4. Cell 95: 379-391.

NIWA, H., BURDON, I., CHAMBERS, I. and SMITH, A. (1998). Self-renewal of pluripotent embryonic stem cells is mediated via activation of STAT3. Genes Dev. 12: 2048-2060.

NIWA, H., MIYAZAKI, J. and SMITH, A.G. (2000). Quanitative expression of Oct$3 / 4$ defines differentiation, dedifferentiation or self-renewal of ES cells. Nat. Genet. 24: 372-376.

OGINO, H. and YASUDA, K. (1998). Induction of lens differentiation by activation of a bZIP transcription factor, L-Maf. Science 280: 115-118.

SMITH, A.G., HEATH, J.K., DONALDSON, D.D., WONG, G.G., MOREAU, J., STAHL, M. and ROGERS, D. (1988). Inhibition of pluripotential embryonic stem cell differentiation purified polypeptides. Nature 336: 688-690.

URA, H., USUDA, M., KINOSHITA,K., SUN, C., MORI, K., AKAGI, T., MATSUDA, T., KOIDE, H. and YOKOTA, T. (2008). STAT3 and Oct-3/4 control histone modification through induction of Eed in embryonic stem cells. J. Biol. Chem.
283: 9713-9723.

WILLEMS, E., MATEIZEL, I., KEMP, C., CAUFFMAN, G., SERMON, K. and LUC LEYNS, L. (2006). Selection of reference genes in mouse embryos and in differentiating human and mouse ES cells. Int. J. Dev. Biol. 50: 627-635.

WILLIAMS, R.L., HILTON, D.J., PEASE, S., WILLSON, T.A., STEWART, C.L., GEARING, D.P., WAGNER, E.F., METCALF, D., NICOLA, N.A. and GOUGH, N.M. (1988). Myeloid leukemia inhibitory factor maintains the developmental potential of embryonic stem cells. Nature 336: 684-687.

YAMAZAKI, Y., KAZIRO, Y. and KOIDE, H. (2001). Ral promotes anchorageindependent growth of a human fibrosarcoma, HT1080. Biochem. Biophys. Res. Commun. 280: 868-873.

YOSHIDA-KOIDE, U., MATSUDA, T., SAIKAWA, K., NAKANUMA, Y., YOKOTA, T., ASASHIMA, M. and KOIDE, H. (2004). Involvement of Ras in extraembryonic endoderm differentiation of embryonic stem cells. Biochem. Biophys. Res. Commun. 313: 475-481.

\section{Further Related Reading, published previously in the Int. J. Dev. Biol.}

See our recent Special Issue Stem Cells and Transgenesis, edited by Robert E. Hammer and Richard R. Behringer at:

http://www.ijdb.ehu.es/web/contents.php?vol=52\&issue =5-6

\section{Recent advances in Drosophila stem cell biology}

John Pearson, Lourdes López-Onieva, Patricia Rojas-Ríos and Acaimo González-Reyes Int. J. Dev. Biol. (2009) 53: 1329-1339 (doi: 10.1387/ijdb.072431jp)

Regulation and function of Spalt proteins during animal development Jose F. de Celis and Rosa Barrio Int. J. Dev. Biol. (2009) 53: 1385-1398 (doi: 10.1387/ijdb.072408jd)

\section{Mouse induced pluripotent stem cells}

Eamon Geoghegan and Lucy Byrnes

Int. J. Dev. Biol. (2008) 52: 1015-1022

Comparative study of mouse and human feeder cells for human embryonic stem cells Livia Eiselleova, Iveta Peterkova, Jakub Neradil, Iva Slaninova, Ales Hamp and Petr Dvorak Int. J. Dev. Biol. (2008) 52: 353-363

Spa-1 regulates the maintenance and differentiation of human embryonic stem cells Young-Jin Lee, Hee-Young Nah, Seok-Ho Hong, Ji-Won Lee, Ilkyung Jeon, Jhang Ho Pak, Joo-Ryung Huh, Sung-Hoon Kim, Hee-Dong Chae, Byung-Moon Kang, Chul Geun Kim and Chung-Hoon Kim

Int. J. Dev. Biol. (2008) 52: 43-53 